FERRET Carole - 2013 «Leurrer la nature : quelques exemples de manipulation des bêtes en Asie intérieure ", Cabiers d'anthropologie sociale $\mathrm{n}^{\circ} 9$ [Leurrer la nature, numéro dirigé par Hélène ARTAUD], p. 72-96.

\title{
Leurrer la nature : Quelques exemples de manipulation des bêtes en Asie intérieure
}

Carole FERRET

Abuser la nature de sorte qu'elle vous donne ce que vous souhaitez, voilà qui est fort tentant. Le leurre correspond à une forme d'action bien particulière, une manipulation consistant à faire croire pour faire agir. Les peuples pasteurs de Sibérie et d'Asie centrale pratiquent des élevages de type extensif, où les animaux participent de diverses manières à leur exploitation. Dans quelle mesure certaines techniques pastorales s'appuient-elles sur le leurre? Adoptant l'approche d'une anthropologie de l'action, nous verrons si les pasteurs iakoutes ou kazakhs leurrent effectivement leur bétail lorsqu'ils les manipulent à l'occasion de l'allaitement ou de la conduite des troupeaux par des huchements.

\section{FAIRE CROIRE POUR FAIRE AGIR}

En Asie centrale, les Kazakhs, pasteurs nomades jusqu'à la collectivisation des années 1930, élèvent des chevaux, des moutons, des chèvres, des vaches et des chameaux dans un milieu de steppes et de déserts. En Sibérie, les Iakoutes, pasteurs semi-sédentaires, élèvent des chevaux, des vaches et des rennes dans un milieu de taïga parsemée de clairières. Il s'agit, dans les deux cas, d'élevages extensifs en troupeaux, le nourrissage des bêtes étant principalement fondé sur le pacage, avec une rotation des pâtures qui peut entraîner, chez les hommes qui accompagnent leur bétail, diverses formes de mobilité.

L'élevage iakoute du cheval est singulièrement peu interventionniste. Il se caractérise par une absence de contrôle du cycle reproductif, une surveillance épisodique des troupeaux et un affouragement minime en dépit d'un climat exceptionnellement rigoureux (Ferret, 2006 ; Ferret, 2012). Ces caractéristiques tendent à le rapprocher d'une activité cynégétique, d'autant que la principale activité des éleveurs consiste à chercher leurs animaux, qui pâturent en liberté : plus que des gardiens de troupeaux, ils sont des «chercheurs» de chevaux (Ferret, 2007). Autre point commun avec la chasse, la viande constitue actuellement le premier débouché de cet élevage (Ferret, 2009).

\section{Le leurre des chasseurs}

Si l'élevage est proche de la chasse, observe-t-on, parmi les techniques pastorales, un recours au leurre - qui relève primordialement du domaine cynégétique ? À la différence des éleveurs, les chasseurs ne maitrisent pas les mouvements du gibier que, faute de débusquer, ils peuvent vouloir attirer. Selon la définition du dictionnaire, le leurre est un terme de fauconnerie désignant initialement un morceau de cuir rouge en forme d'oiseau auquel on attache un appât et qui sert à faire revenir le faucon sur le poing ; par extension, ce mot dénote un artifice destiné à tromper. 
Dans la chasse au vol telle qu'elle est encore pratiquée en Asie centrale, le leurre ne correspond pas toujours à une totale duperie, puisque c'est souvent un animal véritable qui sert de leurre, ou d'appelant pour la capture initiale des oiseaux de proie. L'aigle royal par exemple, est pris grâce à un leurre vivant (tel qu'un oiseau mauvais chasseur) ou un leurre mort (tel qu'une dépouille de lièvre ou de renard) avant d'être apprivoisé, dressé puis utilisé pour la chasse au vol (Jacquesson, 2000 : 114). Le leurre est donc ici une authentique proie, qui n'aurait rien pour décevoir l'animal si on lui donnait la possibilité de s'en repaître. D’autres procédés sont aussi utilisés pour s'emparer du futur auxiliaire de chasse : filet, perche-lasso comme pour la capture des chevaux, chaussons de feutre pour ne pas ébruiter sa venue ou recours au feu pour paralyser l'animal d'étonnement (Čormanov, $1906: 8-10)$.

Par la suite, les jeunes rapaces sont encore entraînés avec des leurres, des oiseaux qu'on a privés de la possibilité de fuir en leur ôtant des ailes, afin que le novice se fasse la main sur une proie facile. Les aigles destinés à la chasse aux antilopes saïga sont d'abord confrontés à des chèvres (Čormanov, 1906 : 6). Il n’y a donc pas ici véritablement illusion, visant à «faire prendre des vessies pour des lanternes », mais une simple substitution spécifique destinée à faciliter le dressage.

Cependant le leurre (kaz. dalbaj ${ }^{1}$ ) est aussi une planchette recouverte d'ailes de pigeon, dont le fauconnier se sert pour rappeler son oiseau (Jacquesson, 2000 : 94). Apprendre à revenir au poing est une partie importante du dressage, qui s'aide d'un autre type de leurre, cette fois réellement décevant, au sens archaïque du terme. Ces divers exemples, tirés du domaine primordial du leurre, la chasse au vol, incitent à s'interroger sur la nature du leurre, suivant que l'illusion qu'il créé est plus ou moins proche de la réalité.

\section{p.74 Une forme particulière de manipulation}

Leurrer est un bel exemple de manipulation, à savoir une action sur un être vivant visant à lui faire faire quelque chose. Tandis qu'une opération est une simple action d'un sujet sur un objet, la manipulation est une action exercée sur un être vivant, lui-même doté d'une capacité d'agir, qui réalise l'action à la place du sujet. Le leurre est un cas particulier de manipulation, où il s'agit de «faire croire pour faire agir». L'action du sujet consiste à travestir un artefact (qui peut être indifféremment inerte ou vivant, voire le sujet lui-même) afin que l'objet, percevant cet artefact, soit abusé par cette perception trompeuse et incité à agir dans le sens désiré.

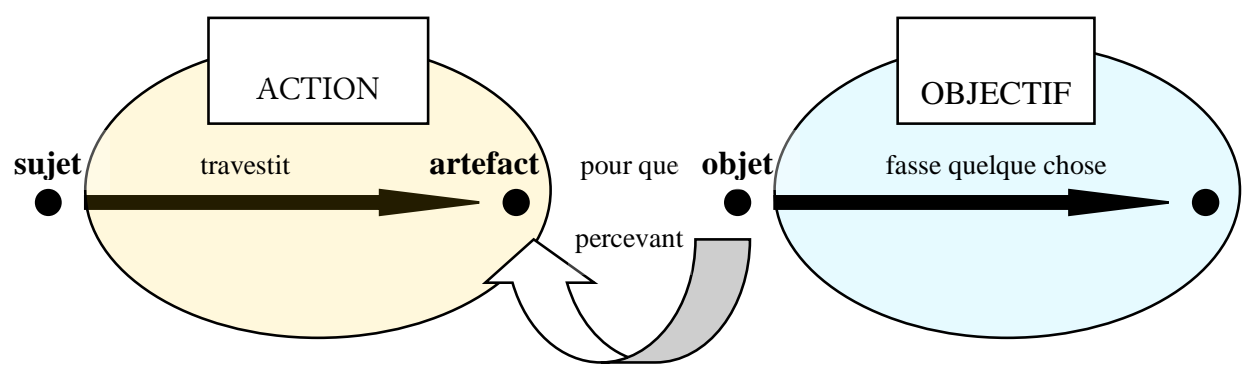

Leurre : faire croire pour faire agir

Leurrer est donc une action complexe qui compte au moins trois actants : un sujet leurrant, un artefact servant de leurre et un objet leurré. Son mécanisme implique que le sujet leurrant suppose une certaine forme d'intentionnalité chez l'objet leurré qui, de lui-même, n'aurait vraisemblablement pas effectué l'action requise si sa perception sensorielle n'avait pas été abusée. En effet, l'efficacité de la manipulation se fonde ici sur une tromperie des sens (vision, ouïe, toucher, goût, odorat ou une combinaison de plusieurs de ces sens). Et, dans le cas d'une communication interspécifique, le sujet tient éventuellement compte du fait que, chez l'espèce animale à laquelle il s'adresse, les capacités sensorielles sont différentes des siennes. 
Le leurre est essentiellement une action indirecte, qui n'atteint pas d'emblée son objectif; une action externe, qui n'agit sur l'objet que par l'intermédiaire d'un artifice tiers; positive ou négative, selon qu'elle attire ou repousse, qu'elle incite à faire telle action ou en dissuade ${ }^{2}$.

Les peuples pasteurs tirent profit de l'élevage à la fois épisodiquement, par la viande du bétail abattu, et quotidiennement, par la traite des femelles allaitantes. Plusieurs procédés sont utilisés pour assurer la continuité de l'allaitement et de la traite, parfois menacée en cas de refus de la mère ou de disparition du petit. Il s'agit alors de persuader une femelle à donner son lait, soit aux hommes, soit à un petit qu'elle refuse de nourrir - le sien ou un autre.

\section{La traite comme manipulation}

\section{La technique « de la sucée»}

En Asie intérieure, la grande majorité des femelles, toutes espèces confondues, est traite par la méthode dite "de la sucée », avec la participation du petit ${ }^{3}$. Au moment de la traite, le veau, le poulain ou le chamelon est amené près de sa mère et tète quelques instants puis, très rapidement, quelqu'un interrompt son repas à peine entamé, l'écarte de la mamelle et le tient à proximité de sa mère pendant la mulsion. Cette sucée préalable du petit a pour but de favoriser la montée de lait. Elle est souvent jugée indispensable : "sans sucée, la vache ne donnera pas son lait », disent les éleveurs. Et elle est même parfois renouvelée en cours d'opération, lorsque le trayeur juge nécessaire de « réamorcer la pompe ${ }^{4}$.
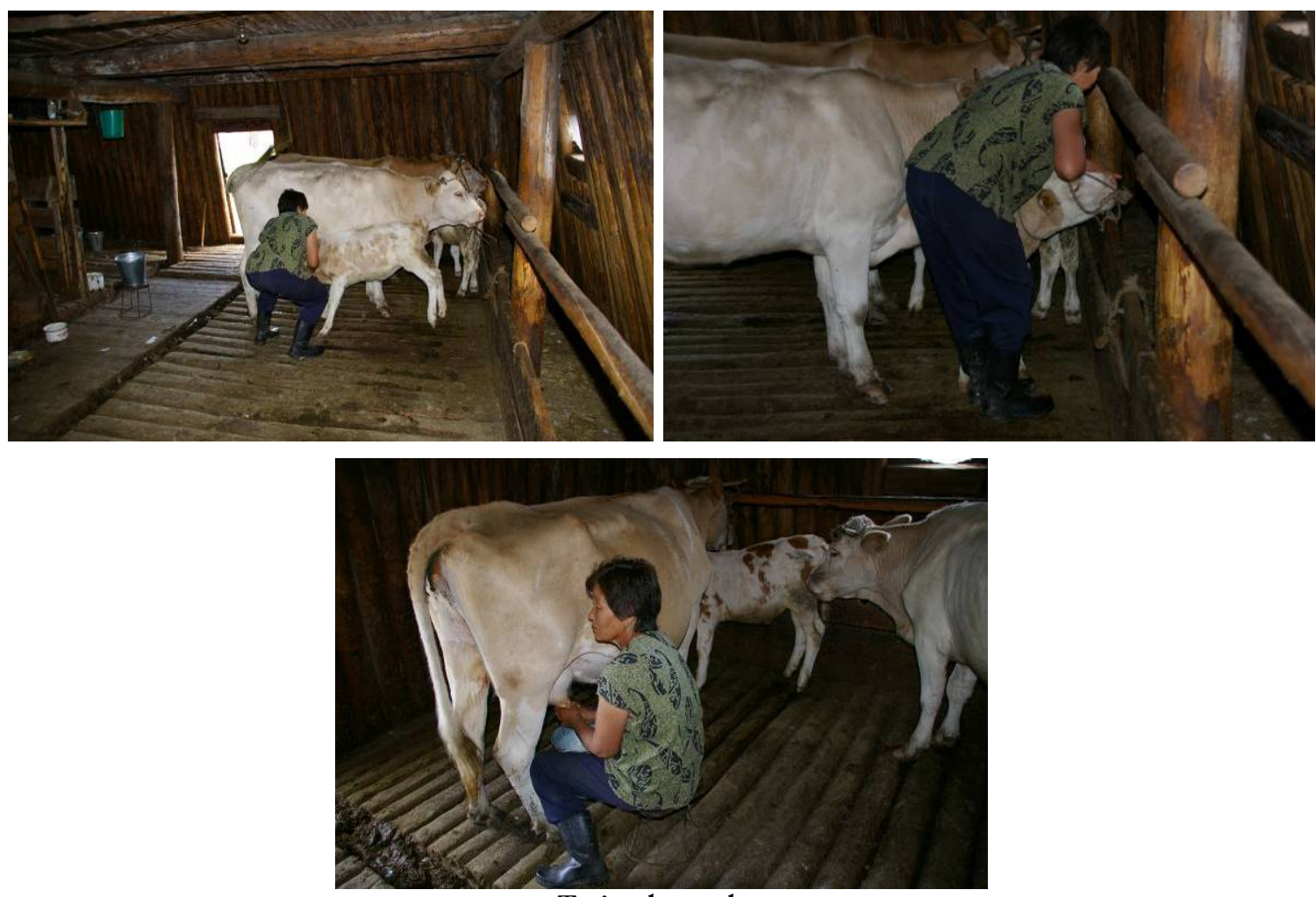

Traite des vaches

dans le iak. hoton " étable », après la sucée du veau ensuite attaché devant sa mère.

Photographies C.Ferret. Iakoutie, ulus d'Ust'-Aldan, août 2008 
Ce procédé est aussi utilisé ailleurs : les Nuers usent du même stratagème pour traire leurs vaches (Evans-Pritchard 1994 [1937] : 40). Plus près de nous, dans le Cantal, la vache Salers se trait toujours avec la participation du veau, qui amorce et achève le processus, le trayeur lui laissant le quatrième trayon (Lienard \& al., 2002). Chez les Mongols, il a été décrit dès l'époque médiévale (Rubrouck, 1985 [1767] : 95).

Aujourd'hui, sur les alpages centrasiatiques, la première capture des poulains est un moment festif, qui réunit au mois de juin un groupe de voisins et d'apparentés. Les hommes attrapent les poulains de l'année, alors âgés d'environ un mois, et ils les attachent au kir. žele (kaz. želi), une corde basse tendue entre deux piquets, où sont régulièrement disposés de petits licols. Le žele sert à la fois à empêcher les poulains de téter, à les garder à la même place et, de ce fait, à limiter aussi les déplacements des juments, qui ne s'éloignent pas volontiers de leur petit. Au moment de la traite, le poulain est momentanément libéré, il avale goulûment quelques gorgées avant d'être arraché à la mamelle et tenu près de sa mère pendant qu'on la trait. La nuit, les poulains sont laissés libres avec leur mère.
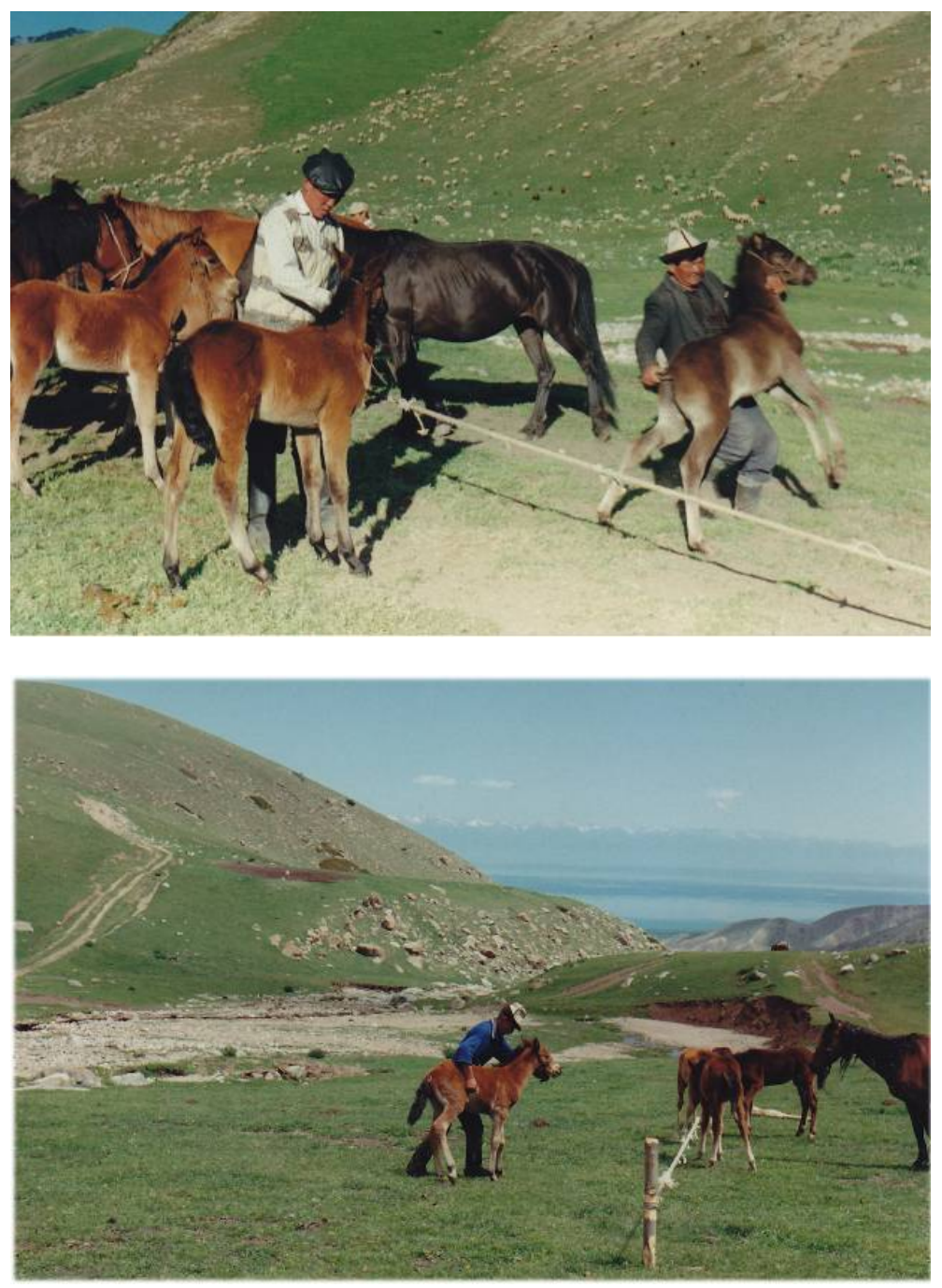

Capture des poulains pour la première traite Photographie C.Ferret. Kirghizstan, région de l'Issyk-koul, juin 1994 


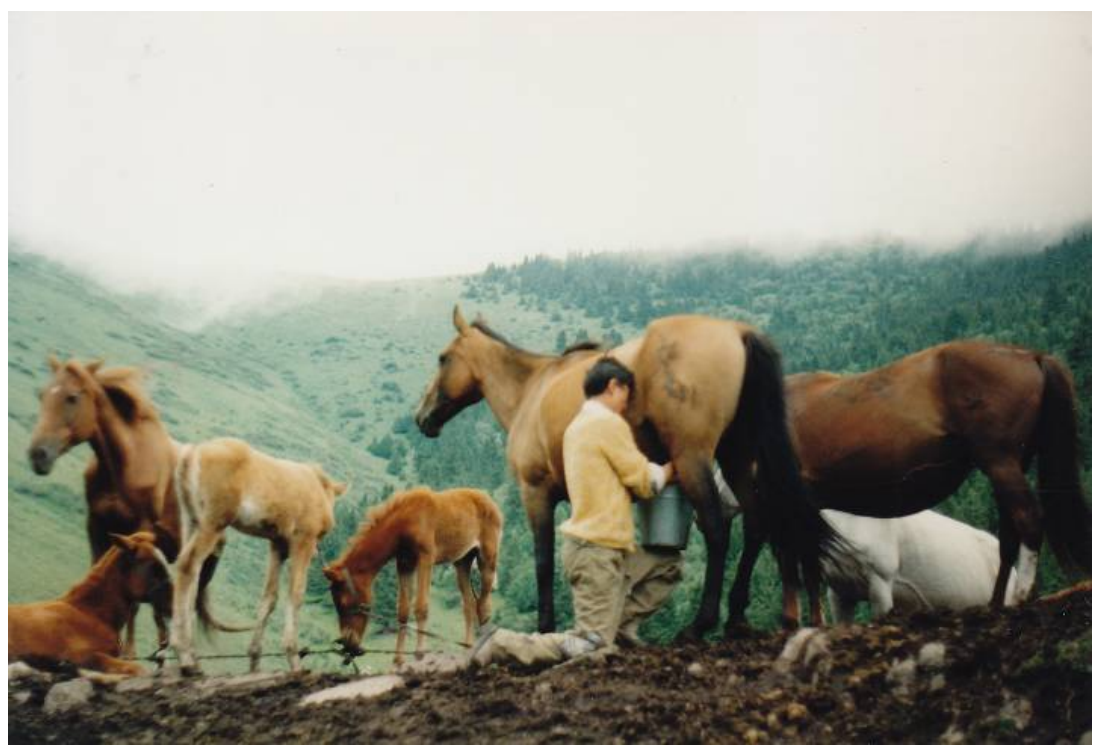

Traite par un jeune éleveur de chevaux près du poulain attaché au želì Photographie C.Ferret. Kazakhstan, région d'Almaty, district de Rajymbek, juillet 1994.

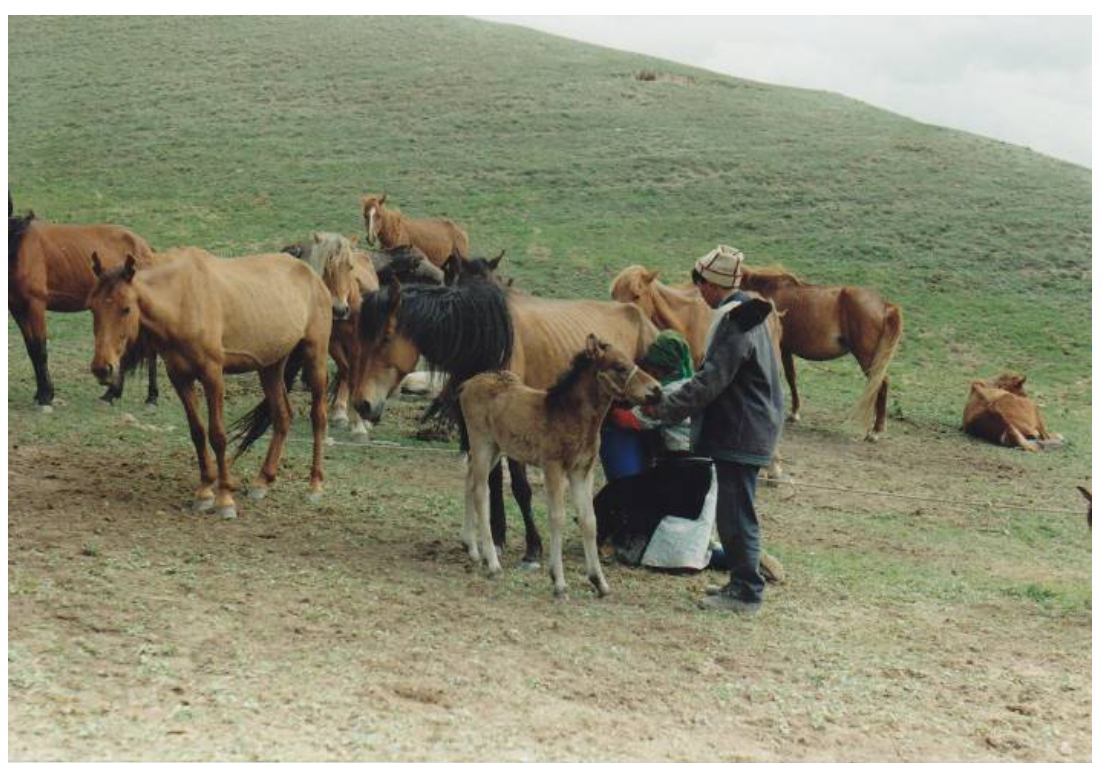

Traite par une femme, tandis qu'un garçon tient le poulain devant la jument Photographie C.Ferret. Kirghizstan, région de Naryn, mai 1994.

Les juments sont traites quatre à huit fois par jour, principalement en été, par des hommes ou par des femmes, qui se tiennent à leur gauche, le genou droit à terre. Le lait de jument, longuement battu, fermente et se transforme en kumys, une boisson légèrement alcoolisée très appréciée. Les vaches sont traites tout au long de l'année, jusqu'au mois précédent le vêlage suivant, deux fois par jour, de préférence par des femmes assises à leur droite sur un petit tabouret.

La traite n'est donc pas une action à deux actants, un sujet trayeur et un objet trait, mais une action qui compte au moins trois actants, avec la participation du petit, dont la présence incite la mère à ne pas retenir son lait. Elle est conçue comme une manipulation, et non comme une simple opération de collecte - telle que le sont, par exemple, la tonte des moutons ou la coupe des crins des chevaux. De manière générale, les pasteurs insistent sur l'aspect participatif de la traite; ils sollicitent le consentement des femelles, jugé indispensable à l'efficacité de cette action.

Aussi la technique de la sucée peut être considérée comme un leurre, faisant croire à la femelle que c'est son petit qui la tète. Qu'importe si vaches ou juments sont réellement dupes, et confondent effectivement leur veau ou leur poulain avec le trayeur. En tout état de cause, la sucée permet, du point de vue des hommes, de créer et d'entretenir cette confusion. 


\section{Autres manières de traire}

Sous le régime soviétique, la traite a été en partie mécanisée. Symboles du progrès, les trayeuses électriques représentaient un bouleversement non seulement parce qu'elles allégeaient la corvée de la traite, mais aussi parce que la mulsion s'effectuait sans la participation du poulain. Destinées à rendre obsolète la traite manuelle (Barmincev \& al. 1980 : 182, 184), elles demeurèrent néanmoins très minoritaires.

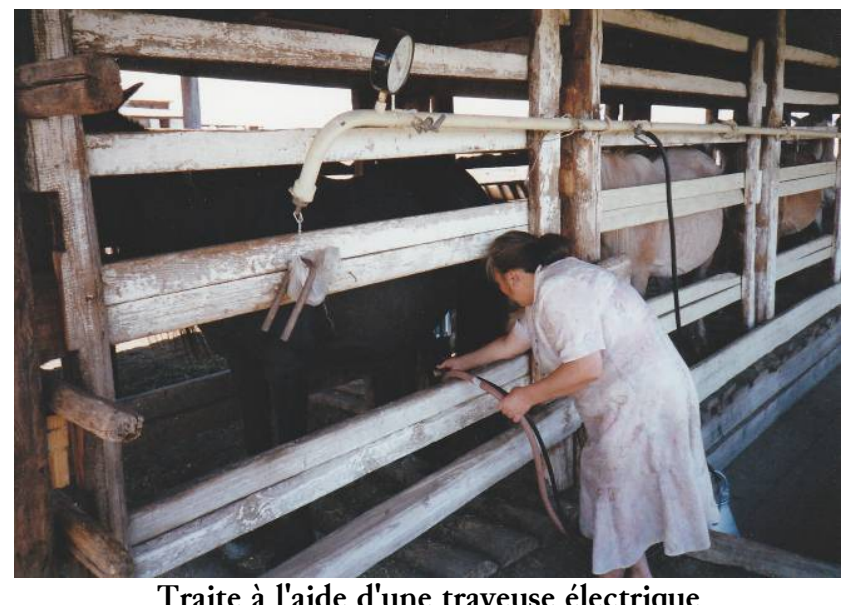

Traite à l'aide d'une trayeuse électrique Ferme à kumys de Pokrovsk

Photographie C.Ferret. Iakoutie, ulus de Hangalas, juillet 1995

La mécanisation représente cependant un changement moins radical qu'il n'y parait. En effet, les trayeurs continuent à juger indispensable le consentement de la femelle, comme en témoignent les paroles d'une employée de la ferme à kumys de Pokrovsk :

«Les premiers jours, les juments s'énervent un peu et ne se laissent pas traire facilement, mais après, elles sont calmes. Elles savent qu'on leur rend leur poulain après la quatrième traite et elles attendent. Les juments comprennent l'homme. Un jour, j'ai poussé une jument un peu brusquement, alors elle ne m'a pas donné de lait à la traite suivante».

En outre, la traite mécanique n'exclut pas toujours le petit : certaines installations permettaient au poulain de demeurer à côté de sa mère, dans une cage placée du côté opposé à la trayeuse. Le poulain, qui avait parfois accès à la mamelle par une ouverture à volet, devait stimuler la lactation par sa présence. Ce dispositif était réservé aux chevaux, car les zootechniciens jugent la technique de la sucée particulièrement utile pour les juments, dont le taux d'ocytocine, principal facteur de la lactation, baisse rapidement ${ }^{5}$.

Par ailleurs, il convient de remarquer que, même lorsque la traite est manuelle, la technique de la sucée n'est pas employée systématiquement. Son utilisation dépend à la fois du nombre de bêtes à traire, des habitudes personnelles des éleveurs et du traitement individualisé des animaux : telle vache aura besoin de la sucée préalable du veau, telle autre pas nécessairement.

D'autres moyens sont employés pour garder les vaches calmes et dociles pendant la traite, notamment à la saison des moustiques, lorsque les pis couverts de boutons sont douloureux. Des entraves postérieures permettent d'éviter les coups de pieds. Face à une vache récalcitrante, sa maîtresse iakoute lui met les doigts dans les naseaux et lui lève le mufle, tandis que, de l'autre main, elle lui prend une corne et lui chante une chanson en citant son nom. Elle caresse souvent ses vaches et évite la présence de tiers, qui risquerait de les inquiéter. Leur gratter les fesses peut aussi les tranquilliser. Enfin un massage du pis préalable à la traite remplace parfois la sucée. Juments et vaches sont donc également traites par la persuasion, dans une action qui relève de la manipulation. Ce sont elles qui donnent leur lait, pas seulement les éleveurs qui le leur prennent. 


\section{Comment convaincre d'adopter}

Il arrive que les hommes doivent se montrer plus persuasifs, lorsque la femelle rejette son propre petit, ou lorsqu'ils veulent la convaincre d'en adopter un autre, dont la mère est morte. Dans ces deux cas, le recours au leurre est parfois manifeste.

\section{Faire accepter son petit}

En Asie intérieure, les éleveurs de chevaux n’interviennent généralement pas dans la mise bas, mais les mères primipares refusent parfois leur petit. Elles ne le reconnaissent pas et ne supportent pas qu'il les tète, compromettant ainsi gravement sa survie. Les éleveurs iakoutes m'ont raconté qu'alors, ils capturent la jument, lui entravent les jambes et la tiennent avant de lui amener le poulain. Parfois, ils commencent par la traire et nourrir le poulain au biberon, avant de le faire téter en veillant à ce qu'elle ne lui envoie pas de coup de pied.

Certains pasteurs centrasiatiques ont recours à des procédés plus élaborés afin d'inciter les femelles récalcitrantes à allaiter leur petit: chant, musique, nouveau-né enduit de sel ou des sécrétions vaginales de sa mère. Ainsi les Touvas attachent la jument et arrosent d'eau salée la croupe du poulain. Une femme âgée, douée d'un don pour apprivoiser les bêtes, se met à chanter en prononçant des voyelles $u$ prolongées : kuru-u-u-g, kuru-u-u-g,kuru-u-u-g,-èt’. Apaisée, la jument finit par lécher le poulain et à le laisser téter (Darža, $2003: 50$ ). Pour les brebis, les paroles different mais le procédé est semblable :

«Il y avait des brebis qui rejetaient leurs agneaux. Cela arrivait certaines années, mais cette fois, c'était aussi contagieux et dévastateur qu'une maladie. À l'heure de la tétée, nous nous accroupissions chacun derrière une brebis et, accrochés à son pis, nous chantions à qui mieux mieux. Oui, nous chantions! Ce n'étaient pas les paroles qui comptaient, mais la mélodie, le son de notre voix et la répétition du mot toega - toega - toega ... [...]

Si tu n'aimes pas ton petit, toega - toega - toega

Tu n'es qu'une chipie, toega - toega - toega

Allons, occupe-t-en, toega - toega - toega [...]

Nous enduisions le derrière de l'agneau avec de la saumure et fourrions le museau de la mère dans sa toison imprégnée de sel. Elle avait beau commencer par se défendre, elle finissait par devoir y goûter en léchant son museau mouillé. Ensuite elle se léchait longtemps, jusqu'à ce que le goût du sel disparaisse complètement. C'est là qu'on voyait ce qui triomphait : l'envie de sel ou l'entêtement [...] On enfonçait plusieurs doigts dans le vagin de la brebis, on les allongeait et les repliait, puis on les essuyait au passage sur le dos de l'agneau; le nombre de doigts et la façon de les replier dépendait des cas. Une brebis pouvait se montrer sensible tandis qu'une autre avait l'air d'une bûche »(Tschinag, 1996 [1994] : 123124).

Les Bouriates d'Aga agissent de même :

«Lorsqu'une femelle refusait d'allaiter, on donnait à téter du lait de vache au petit et on essayait de les "réconcilier". Un chamelon délaissé était reconduit vers sa mère et les éleveurs jouaient d'un instrument à cordes rudimentaire bur, qui rappelle le violon. À défaut, on frappait sur un récipient en fonte avec une patte de mouton ou on faisait chanter une clochette en passant une baguette sur son pourtour. La chamelle ne tardait pas à sangloter profondément, à flairer son petit et à se laisser téter en versant de grosses larmes » (Linhovojn, $1972: 19)$.

Le film de L.Falorni et B.Davaa, Le chameau qui pleure (2003), montre précisément une chamelle se laisser amadouer par la musique en ces circonstances. Si plusieurs de ces techniques sont utilisées dans d'autres parties du monde, notamment en Afrique, le recours à la musique paraît plus original. Est-ce toujours un leurre? En effet, un chamelon n'émet pas de tels sons. Plus que de leurrer, il s'agirait ici d'émouvoir pour éveiller la compassion de la mère animale.

"Thanks to her musical ear and feeling, the camel who first was wildly and furiously staring at her young, soon lets herself be moved to compassion by the sad songs » (Serruys, $1985: 63$ ).

Enfin, à l'inverse de l'apaisement, un sentiment de frayeur peut également être créé et exploité pour provoquer chez la femelle un réflexe de défense du petit et l'obliger à jouer son rôle de mère. 
"Il suffisait parfois de laisser un chien de berger approcher l'agneau abandonné ; l'instinct maternel se réveillait chez la brebis, qui chargeait le chien et s'occupait à nouveau de son petit » (Linhovojn, 1972 : 19) ${ }^{6}$.

\section{Provoquer l'adoption d'un autre petit}

Quand une femelle suitée meurt, les éleveurs s'efforcent de faire adopter son rejeton par une autre mère, pour éviter d'avoir à le nourrir au biberon. De même quand un petit meurt, l'adoption d'un autre jeune permet d'entretenir la lactation de la mère. Les éleveurs emploient alors des techniques proches de celles décrites ci-dessus, destinées à favoriser l'allaitement, ainsi que d'autres plus spécifiques.

Les poulains iakoutes orphelins peuvent être confiés à une autre jument, qui a perdu son poulain, ou une bonne laitière, capable d'allaiter deux petits. Les adoptions spontanées existent, mais elles sont rares. Pour faire accepter ce nouveau poulain à sa nourrice, on la garde attachée une journée sans eau ni nourriture ; puis, le lendemain, on lui présente le poulain juste après l'avoir abreuvée (Gabyšev, 1972 : 306). Ayant constaté qu'une vache qui a perdu son petit donne moins de lait, les Iakoutes s'efforcent de lui faire adopter un autre veau, en couvrant le pupille de la peau du veau mort et en prenant soin de la saler pour que la vache le lèche (Seroševskij, 1993 [1896] : 153-154). Les Kazakhs couvrent également le poulain adoptif de la peau du poulain mort (Dobrosmyslov, 1895 : 96) ou ils l'enduisent régulièrement du lait de la jument, tandis qu'ils brouillent la vue de cette dernière par un mélange de lait et de sel (Baskin, 1982 : 39).

J.Gmelin explique que, pour la poursuite de la traite en cas de disparition du veau, les Bouriates de Transbaïkalie confectionnaient un animal empaillé (1767 [1751-1752], I : 214-215). Le recours à un veau ou, plus fréquemment, à un chameau empaillé est également évoqué en Afrique par C. et M. Le Cœur chez les Daza du Niger (Baroin, 1975 : 493) et par E.Bernus chez les Touaregs. Ce subterfuge est néanmoins plus rare que la substitution par un autre petit, qui offre deux avantages.

p.81 Premièrement il est douteux qu'une femelle puisse être durablement leurrée par un animal empaillé : "la vache, en léchant le mannequin, même recouvert de la peau du veau mort, sent la paille et n'est pas dupe » (Bernus, 1980 : 110). Deuxièmement, outre la poursuite de la traite, l'adoption permet également l'alimentation d'un autre jeune.

Pour persuader une mère d'adopter un deuxième chevreau ou agneau, les Mongols Darkhates tentent de l'amadouer par des paroles ponctuées de l'interrogation rhétorique : "N'est-ce pas ton fils ? », accompagnée des huchements «puëj, puëj!», «tajgoo, tajgoo!», ou «čüü, čüü ! " pour un chevreau. En cas de refus, le chevreau est enduit de sel et de salpêtre (Badamxatan, 1986 : 78).

«Un informateur décrit ainsi une mise en nourrice d'un chamelon dans les années 1920 : tôt le matin, on mouillait et on enduisait de sel et de salpêtre le chamelon, comme s'il venait de naître, et on le maintenait près des mamelles de la chamelle. On psalmodiait alors les paroles appropriées en s'accompagnant d'un instrument à cordes (bjasaanzan xuur) : rapidement, des larmes coulaient des yeux de la chamelle, comme si elle pleurait ; croyant vraiment que c'était son petit, elle le laissait téter» (Badamxatan, $1986: 79$ ).

«Lorsqu'il s'agissait d'un poulain, on le mouillait et on enduisait son dos d'un peu de sel (comme s'il venait de naître), puis on entravait la jument et en psalmodiant "guraj, guraj!”, on l'habituait à laisser téter le poulain. On s'y prenait de la même façon pour un veau, en criant “ööv, ööv! uuv, uuv !” (“æö, xöö" pour femelle de xajnag ou de yack) ; parfois on recouvrait de la dépouille de son veau mort le veau que l'on voulait faire accepter à la mère nourricière » (Badamxatan, 1986 : 79).

Les Iakoutes usaient dans ce cas de procédés stimulant la lactation : ils introduisaient dans le vagin de la jument ou de la vache un bouchon oblong, dit kupčuur, fait d'herbes et de courroies de cuir pliées. L'irritation ainsi causée était censée favoriser la montée de lait. Le kupčuur pouvait aussi être utilisé de manière usuelle pour la traite. En cas d'adoption d'un veau par une mère étrangère, il était laissé dans le vagin pendant une journée, puis passé sur le corps de la vache et du veau (Hudâkov, 1969 : 239 ; Pekarskij, 1907-1930 : 1239). À défaut de kupčuur, on introduisait une main dans le vagin de la vache en disant uo-uo, puis on la lui faisait renifler (Seroševskij, 1993 [1896] : 154 ; Bašarin, 1962 : 97). 


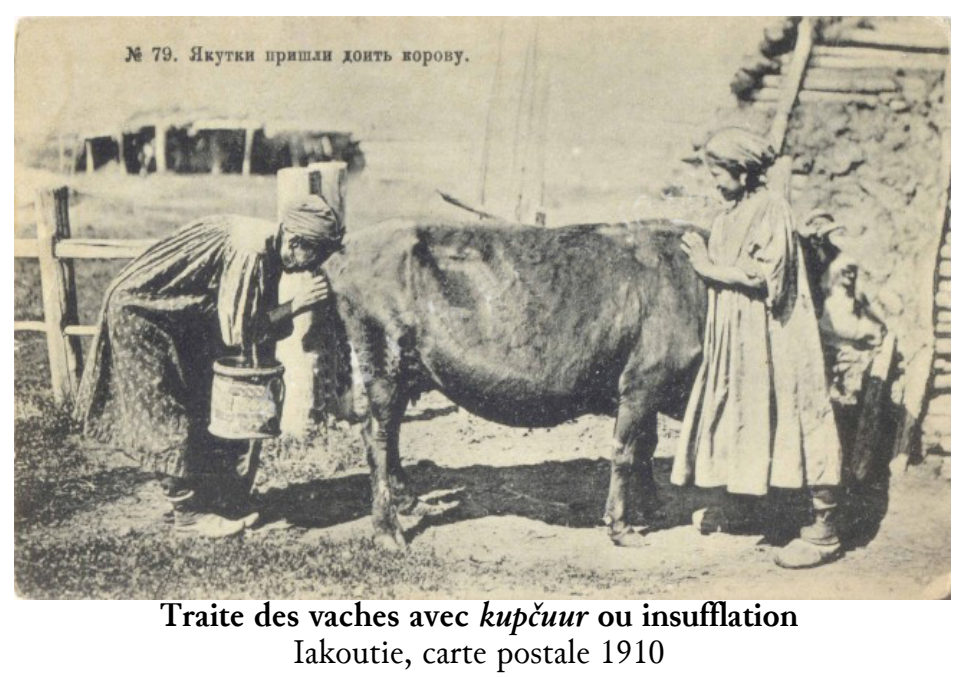

Un autre procédé consistait à souffler dans le vagin en appliquant les lèvres sur la vulve p.82 (Troŝanskij, 1911 : 83 ; Seroševskij, 1993 [1896] : 154). Cette technique d'insufflation accompagnant la traite est attestée de longue date en Afrique saharienne, de l'Est et du Sud, ainsi qu'en Inde, en Europe de l'Est, en Irlande ou en Alsace (Le Quellec, 2011) et sporadiquement en Chine (Huston Edgar, 1924). Chez les Scythes, Hérodote explique que les esclaves voués à la traite des juments :

"prennent des tubes en os fort semblables à des flûtes, ils les introduisent dans les parties sexuelles des juments, puis ils soufflent dans ces tubes et, en même temps, d'autres traient les bêtes. Par ce procédé, disent-ils, l'air gonfle les veines de la bête et le lait descend dans les mamelles » (Hérodote, Enquête IV$2: 358)$.

Selon les interprétations, c'est soit l'irritation du vagin, soit son gonflement, soit encore l'odeur des sécrétions vaginales qui a pour effet de stimuler la lactation. Cette technique peut aussi être vue comme un leurre, dans la mesure où elle fait croire à la femelle qu'elle vient de mettre bas. Chez les Bédouins, les Touaregs et les Daza du Niger, les narines et l'anus de la chamelle adoptante pouvaient être temporairement suturés, afin de bloquer l'olfaction et de donner l'impression d'une mise bas par une volumineuse défécation, une fois l'anus libéré (Baroin, 1975 : 494-495 ; Bernus, 2002 : 404407) ${ }^{7}$.

Le caractère manipulatoire de la traite et la quête du consentement de la femelle ne sont donc pas toujours synonymes de bon traitement. De la même manière qu'en Inde, Gandhi condamnait cet expédient (Burgat, $2004: 237$ ), les membres d'une expédition soviétique, menée dans les années 1920 pour étudier les conditions de l'élevage en Iakoutie, accusent le douloureux kupčuur d'être responsable de la grande nervosité des vaches iakoutes (Šubskaâ \& Saltykov, $1931: 20$ ).

\section{L'adoption est-elle un leurre ?}

Les techniques utilisées pour favoriser l'adoption poursuivent donc les objectifs suivants : 1) faire croire à la femelle que le pupille est son propre petit (en le recouvrant de la peau du jeune mort, en l'enduisant des sécrétions vaginales de la femelle) ; 2) donner l'illusion qu'elle vient de mettre bas (par l'insufflation, en ôtant le kupčuur, en mouillant et salant le petit, en imitant les pleurs du nouveau-né) ; 3) l'empêcher de reconnaître l'intrus (en lui brouillant la vision et l'olfaction) ; 4) l'inciter à le lécher pour créer un attachement (en mettant du sel sur le dos du petit) ; 5) stimuler un instinct maternel de défense (en provoquant l'attaque d'un chien). Toutes ces actions peuvent être assimilées à des leurres, dans la mesure où elles illusionnent pour faire agir (ou ne pas agir). Les actions qui font croire à la femelle qu'elle vient de mettre bas ou que c'est son petit qui la tète peuvent être qualifiées de positives; celles qui l'empêchent de reconnaître l'intrus sont négatives. 
Cependant, d'autres stratagèmes sont employés, qui ne correspondent pas à des leurres : entraver la femelle pour l'empêcher de taper ; l'abreuver pour que la tétée soulage ses mamelles gonflées.

Deux stratégies opposées, consistant soit à apaiser soit à effrayer la femelle, peuvent concourir au même objectif. Ainsi le fait de provoquer un réflexe de défense par l'attaque d'un chien peut être interprété comme une manipulation que je qualifierais de contraire. À l'inverse des huchements et des mélodies qui calment et rassurent, l'intervention du chien peut sembler surprenante puisqu'elle va à l'encontre de l'apaisement, mais c'est là un geste tactique qui consiste à soumettre le petit à un danger pour inciter la femelle à le protéger et donc à l'adopter.

On distinguera enfin plusieurs actions de type interne, agissant sur le corps des animaux (abreuvement, entrave, kupčuur, insufflation, eau, sel, lait, sécrétions vaginales), de quelques actions externes (musique, chien). Toutes ces techniques font appel à un vaste éventail de sensations et font intervenir tous les sens de l'animal (vue, toucher, odorat, goût, ouïe). Parmi les procédés auditifs, des onomatopées attribuées en propre à chaque espèce se retrouvent aussi bien dans les chants incitant à l'allaitement que dans les cris de conduite des troupeaux ${ }^{8}$. Nous allons à présent nous pencher sur ces derniers, afin de voir si ces huchements relèvent du leurre.

\section{EST-CE LEURRER QUE DE HUCHER ?}

\section{Les huchements des pâtres d'Asie intérieure}

En Asie centrale, les pasteurs dirigent leur bétail à la voix ${ }^{9}$. Pour rassembler un troupeau, le faire venir ou le pousser, les éleveurs émettent des huchements, définis par R. Dor comme «un interpellatif (vocal ou sifflé) adressé par l'homme à l'animal domestique pour influer sur son comportement» (1995: 201). Ce même auteur distingue les vocalisations somatotropes «faire approcher la proie pour la capturer», somatofuges "faire fuir le prédateur» et somatoneutres " neutraliser le mouvement de l'animal pour qu'il reste à distance constante de son maitre » (Dor, $2002: 131-132)$.

p.84 Il existe un grand choix de huchements, qui varient suivant l'espèce concernée, mais se retrouvent aussi d'une ethnie à l'autre, dans plusieurs variantes correspondant à un modèle commun. Quelques-uns ont été lexicalisés, transformés en verbes ou en substantifs désignant une espèce animale.

Huchements iakoutes ${ }^{10}$

\begin{tabular}{|c|c|c|c|c|c|}
\hline & $\begin{array}{l}\text { faire venir un } \\
\text { troupeau }\end{array}$ & $\begin{array}{l}\text { faire avancer un troupeau, faire } \\
\text { fuir, repousser }\end{array}$ & $\begin{array}{c}\text { faire avancer ou accélérer sa } \\
\text { monture }\end{array}$ & $\begin{array}{c}\text { calmer sa } \\
\text { monture, ralentir }\end{array}$ & $\begin{array}{l}\text { arrêter sa } \\
\text { monture }\end{array}$ \\
\hline chevaux & $\begin{array}{l}\text { kuru; } \\
\text { boruu; } \\
\text { kèliy “venez" }\end{array}$ & $s a-a-a t$ & $\begin{array}{l}\text { hot; bè ; } \\
\check{c} u ; \\
\text { aj; bö ; baj; byi ; byjda; } \\
\text { claquement de langue ; } \\
\text { čè [au départ] ; } \\
\text { trrrr ou tprr [pour faire } \\
\text { trotter] ; } \\
\text { buss ou sss [pour pousser à la } \\
\text { vitesse maximale] }\end{array}$ & $b a$ & $\begin{array}{l}\text { brrr; } \\
\text { prrr; } \\
\text { trrr }\end{array}$ \\
\hline rennes & $m \grave{e}$ & $\begin{array}{l}\text { aba; } \\
\text { brrr; } \\
\text { trrr; } \\
\text { divers cris aigus et sifflements }\end{array}$ & & & \\
\hline
\end{tabular}


Huchements kazakhs ${ }^{11}$

\begin{tabular}{|c|c|c|c|c|c|c|c|c|c|c|}
\hline & $\begin{array}{c}\text { faire venir à } \\
\text { soi }\end{array}$ & $\begin{array}{l}\text { faire } \\
\text { avancer, } \\
\text { faire fuir, } \\
\text { repousser }\end{array}$ & capturer & $\begin{array}{l}\text { faire } \\
\text { boire }\end{array}$ & $\begin{array}{c}\text { faire } \\
\text { s'accou- } \\
\text { pler }\end{array}$ & $\begin{array}{l}\text { faire le- } \\
\text { ver et } \\
\text { avancer }\end{array}$ & $\begin{array}{l}\text { faire } \\
\text { bara- } \\
\text { quer }\end{array}$ & $\begin{array}{l}\text { calmer, } \\
\text { deman- } \\
\text { der im- } \\
\text { mobilité }\end{array}$ & $\begin{array}{c}\text { faire } \\
\text { accélérer } \\
\text { monture }\end{array}$ & $\begin{array}{c}\text { faire } \\
\text { ralentir } \\
\text { mon- } \\
\text { ture }\end{array}$ \\
\hline chevaux & $\begin{array}{l}\text { qyru [s'ils } \\
\text { sont loin] ; } \\
\text { mo [s'ils } \\
\text { sont près] }\end{array}$ & $\begin{array}{l}a j \\
\check{c} \ddot{O}\end{array}$ & $q r$ & ǩse & qurajt & & & $\begin{array}{l}\text { oj; } \\
\text { tèk; } \\
\text { claque- } \\
\text { ment de } \\
\text { langue }\end{array}$ & $\begin{array}{l}\check{c} u ; \\
\check{s} \ddot{u}\end{array}$ & $d r r r$ \\
\hline moutons & $\begin{array}{l}\text { tprruaj; } \\
\text { pсӧ }\end{array}$ & $\begin{array}{l}\text { rrbajt; } \\
\text { qos }\end{array}$ & $q o s ̌$ & quraj & $\begin{array}{l}\text { qožuq; } \\
\text { qoš }\end{array}$ & & & & & \\
\hline chèvres & $\begin{array}{l}\text { šure } \\
\text { šörej }\end{array}$ & $\begin{array}{l}\text { šig; } \\
d z ̌ i k\end{array}$ & šege & šaum & & & & & & \\
\hline vaches & $\begin{array}{l}\text { aubau; } \\
\text { mo }\end{array}$ & $a q q$ & auqum & šaum & $g \check{z}$ & & & & & \\
\hline chameaux & kös & & & sorap & qurajt & $\check{s} \mathcal{u}$ & šök & & & \\
\hline ânes & & & & & & & & & $b \ddot{~}$ & $\ddot{u} \check{s}$ \\
\hline chiens & $k \ddot{u} \check{s}^{\prime}$ & ket & & & & & & & & \\
\hline chats & $p \check{s}$ & & & & & & & & & \\
\hline
\end{tabular}

Huchements touvas ${ }^{12}$

\begin{tabular}{|c|c|c|c|c|c|c|c|}
\hline & $\begin{array}{l}\text { faire venir à } \\
\text { soi }\end{array}$ & $\begin{array}{l}\text { faire venir } \\
\text { un petit } \\
\text { isolé vers le } \\
\text { groupe }\end{array}$ & $\begin{array}{l}\text { faire } \\
\text { avancer } \\
\text { faire fuir, } \\
\text { repousser }\end{array}$ & calmer, apaiser & $\begin{array}{l}\text { calmer pendant le } \\
\text { harnachement, } \\
\text { demander im- } \\
\text { mobilité }\end{array}$ & faire baraquer & $\begin{array}{l}\text { faire } \\
\text { lever }\end{array}$ \\
\hline chevaux & $t p r-\hat{s} O$ & kuru-u-u-g & $\check{s} u$ & $\begin{array}{l}\text { kuru-u-u-g, } \\
k u r u-u-u-g-\grave{e} t\end{array}$ & $t p r-u$ & & \\
\hline moutons & $\begin{array}{l}\text { toopta- } \\
\text { toopta } \\
\text { tprot, } \\
\text { tprot, } \\
\text { tprot }\end{array}$ & & $\hat{s}-\hat{s}-\hat{s}-\hat{s} i$ & & bolba & & \\
\hline chèvres & $\begin{array}{l}\check{c} u-\check{c} u ; \\
\check{c} i-c ̌ i \text { [pour } \\
\text { les } \\
\text { chevreaux] }\end{array}$ & & dajgy & $\check{s} \ddot{0}$ & $\check{s} \ddot{s}$ & & \\
\hline vaches & & & bo- $\stackrel{c}{u}$ & $b \ddot{o}-\ddot{g} g$ & $b a$ & & \\
\hline chameaux & & & & & & $\begin{array}{l}\text { sok [en tapant } \\
\text { du doigt sur un } \\
\text { antérieur] }\end{array}$ & bok \\
\hline
\end{tabular}


Huchements mongols ${ }^{13}$

\begin{tabular}{|c|c|c|c|c|c|c|c|c|c|}
\hline & $\begin{array}{l}\text { rappeler } \\
\text { des } \\
\text { animaux } \\
\text { qui } \\
\text { s'éloignent }\end{array}$ & $\begin{array}{l}\text { faire avancer ou } \\
\text { accélérer un } \\
\text { troupeau }\end{array}$ & $\begin{array}{l}\text { faire } \\
\text { chan- } \\
\text { ger de } \\
\text { di- } \\
\text { rection }\end{array}$ & $\begin{array}{l}\text { faire arrêter, } \\
\text { ralentir }\end{array}$ & $\begin{array}{l}\text { attraper } \\
\text { jeunes }\end{array}$ & $\begin{array}{l}\text { apaiser pour } \\
\text { traite ou } \\
\text { allaitement }\end{array}$ & $\begin{array}{c}\text { faire } \\
\text { baraquer }\end{array}$ & $\begin{array}{l}\text { deman- } \\
\text { der im- } \\
\text { mobilité }\end{array}$ & $\begin{array}{l}\text { faire avancer, } \\
\text { accélérer } \\
\text { monture ou } \\
\text { animal attelé }\end{array}$ \\
\hline chevaux & $\begin{array}{l}\text { gurui; } \\
\text { burae }\end{array}$ & $\begin{array}{l}\text { büdi; } \\
\text { baja; } \\
\text { bae; } \\
\dot{g} u ; \text { sifflements ; } \\
\dot{e} e ̀, c \check{c} u\end{array}$ & baj & $\begin{array}{l}\text { baj [pour un } \\
\text { cheval } \\
\text { isolé] ; budg } \\
\text { [pour un } \\
\text { troupeau, } \\
\text { précédé d'un } \\
\text { sifflement] ; } \\
\text { brrr, prrr }\end{array}$ & dzèlè & $\begin{array}{l}\text { baja; } \\
\text { bae; } \\
\text { ba ; } \\
\text { bas ; } \\
\text { guri ; } \\
\text { gurii }\end{array}$ & & $\begin{array}{l}\text { buš ; } \\
\text { boš ; } \\
\text { baj }\end{array}$ & $\check{c} u$ \\
\hline moutons & tfffe & $\begin{array}{l}\text { čae; } \\
\text { tadi; čaga; } \\
\text { döči, išš̈ [pour } \\
\text { ébranler un } \\
\text { troupeau de } \\
\text { moutons au } \\
\text { repos] ; } \\
\text { baj [précédé } \\
\text { d'un sifflement] }\end{array}$ & baj & & $\begin{array}{l}\text { högönö; } \\
\text { gürgi }\end{array}$ & $\begin{array}{l}\text { dae; } \\
\text { čae; } \\
\text { bolbae, } \\
\text { bolboe; } \\
\text { toeġo; } \\
\text { tojiego ; } \\
\text { taagü ; } \\
\text { bos }\end{array}$ & & & \\
\hline chèvres & $\begin{array}{l}\check{c} u \\
d \check{z} u \\
\check{s} u\end{array}$ & $\check{c}$ čaja & $\check{c} \ddot{o}, \check{c} a$ & & $\begin{array}{l}\text { bögönö; } \\
\text { gürgi }\end{array}$ & $\begin{array}{l}\text { dae ; ̌́ae; } \\
\text { ičige, čige ; } \\
\text { čegeg ; zuu }\end{array}$ & & & \\
\hline vaches & $\begin{array}{l}k \ddot{o w} ; \\
\ddot{o} w ; \ddot{o}\end{array}$ & $\begin{array}{l}\text { böči ; bödži ; } \\
\text { bög; hog; } \\
\text { bo [précédé d'un } \\
\text { sifflement] ; } \\
\text { budg [pour une } \\
\text { seule bête] }\end{array}$ & & & $\begin{array}{l}d z e ̀ l e ̀ ; \\
\text { gürgi; } \\
\text { zel [faire } \\
\text { aller un } \\
\text { jeune } \\
\text { dans un } \\
\text { enclos } \\
\text { pour la } \\
\text { traite] }\end{array}$ & $\begin{array}{l}\text { bae, baja, } \\
\text { ba; ö̈b; } \\
\text { ö̈; } \\
\text { suug [pour un } \\
\text { yak] }\end{array}$ & & $\begin{array}{l}\text { baa; } \\
\text { drrr }\end{array}$ & $\begin{array}{l}\text { budg [pour } \\
\text { un } \\
\text { animal] ; } \\
\text { bo, bog } \\
\text { [pour plu- } \\
\text { sieurs] ; bo }\end{array}$ \\
\hline chameaux & $\begin{array}{l}\text { dür; } \\
\text { gür ; } \\
\text { bös ; tüs; } \\
\text { tor }\end{array}$ & $\begin{array}{l}\text { böči } \\
\text { bög tsü }\end{array}$ & & & $\begin{array}{l}\text { tor; } \\
\text { dur }\end{array}$ & $\begin{array}{l}\text { dur; duur; } \\
\text { ba; bo }\end{array}$ & sög ; čok & & \\
\hline
\end{tabular}

Sans analyser ici tous ces huchements dans le détail, nous remarquerons qu'en dépit de leur variété, il n'existe pas un et un seul huchement par espèce et par commande, puisque la même vocalisation peut s'employer pour plusieurs espèces - subissant alors une "expansion ", au sens où l'entend R.Dor (1982 : 20) - et aussi parfois pour des ordres différents, voire opposés, tantôt stimulant, tantôt apaisant, suivant une « déviation sémantique » qui paraît plus surprenante.

\section{Expressifs, impressifs ou injonctifs}

Dans la perspective où certains huchements d'appel sont parfois interprétés comme une imitation du cri animal, ils pourraient être considérés comme des leurres, à l'instar des appeaux des chasseurs. Ces huchements qui ressemblent au cri animal sont qualifiés d'expressifs par R.Dor, qui distingue quatre types définis ainsi : 
«Les huchements expressifs reproduisent non pas vraiment la vocalisation de l'animal, mais la sensation phonique de cette vocalisation. C'est pourquoi il peut y avoir une certaine variabilité, mais cependant ils changent peu d'une langue à l'autre. Les huchements impressifs sont les plus nombreux, ils proviennent de l'observation de l'efficacité de certains sons sur les animaux qui les perçoivent plus clairement et y réagissent mieux. La troisième catégorie [huchements descriptifs] regroupe les huchements qui font référence au nom de l'espèce ou de l'individu, à un trait physique ou comportemental. Il peut s'agir de noms qui subissent des déformations importantes. Ce sont les moins nombreux et ceux qui présentent le particularisme linguistique le plus marqué. La dernière catégorie de huchements [injonctifs] est un peu moins homogène : elle comprend des impératifs (surtout de "venir"), des adverbes de lieu et des syntagmes prépositionnels» (1993:32-33. C’est moi qui souligne).

Kuru, huchement d'appel des chevaux partagé par de nombreux peuples turcs (Dor, 1993 : 3436 ; Ferret, 2006 : 264-267, 574-577 ; tableaux supra), souvent interprété comme l'imitation d'un hennissement, est donc un huchement expressif. Les gardiens de troupeaux appelleraient donc les chevaux en imitant le cri de leurs congénères. Certains éleveurs préfèrent cependant employer un huchement de type injonctif, plus proche du langage humain.

«Pour appeler le troupeau, habituellement, on fait kuru, kuru, kuru, mais moi, ça ne me plait pas. Moi

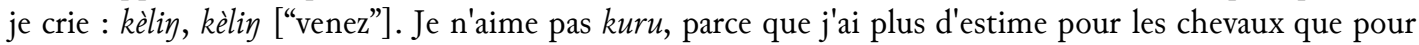
les autres animaux. Il faut parler aux chevaux avec des mots, comme à des hommes [...] et pas seulement faire du bruit» ${ }^{14}$.

Le caractère expressif ${ }^{15}$ est surtout présent dans les huchements d'appel, qui correspondent donc à la formule «imiter le cri pour faire venir». E.Maj remarque, par exemple, que les Iakoutes appellent les troupeaux de rennes par un cri nasal mè, mè, mè, imité des bruits émis par les faons et leur mère, lorsqu'ils se perdent de vue ou se retrouvent dans des troupeaux différents (2007 : 166167).

D'autres huchements d'inspiration mimétique s'éloignent de ce modèle du leurre et sont de type impressif, provoquant une réaction de l'animal par l'évocation sonore d'un geste. C'est le cas du sifflement pour l'abreuvement ${ }^{16}$ ou du kirghize pamiri šorop incitant le chameau à boire, qui ressemble au bruit de l'animal qui lampe (Dor, 1995 : 217). Il ne s'agit plus ici d'appeler en faisant croire à la présence d'un congénère, mais plutôt de donner envie de boire en reproduisant ce bruit. Plus nombreux et plus fréquents, les huchements impressifs influent sur le comportement animal par leur action stimulante ou apaisante.

\section{L'action des huchements}

En tant que signes de la communication interspécifique, les huchements se distinguent des signes linguistiques de la communication intraspécifique par leur moindre part d'arbitraire. S'ils possèdent un aspect conventionnel, d'où leur variabilité, on ne peut leur dénier toute motivation. Ce n'est pas un hasard si les huchements stimulants tels que bue ou $\check{c} u$ se disent de manière brève et répétée, sur un ton énergique, tandis que la trille brrrr est prolongée. Comme les onomatopées dans le langage humain, les huchements sont à la fois conventionnels et motivés. Tout en différant selon les langues (cocorico, kukareku, cock-a-doodle-to, etc.) ${ }^{17}$, les onomatopées exprimant les cris d'animaux se ressemblent entre elles, comme elles ressemblent aux bruits émis par ces animaux ${ }^{18}$. De même, les huchements utilisés pour conduire les bêtes se ressemblent et entretiennent souvent un lien qui tend à être direct et positif - parfois suffisant mais jamais nécessaire - avec les réactions qu'elles entendent provoquer (approche ou fuite, calme ou excitation).

Les huchements permettant de calmer une monture qui s'agite ou de ralentir son allure sont souvent les mêmes, mais leur action ne peut être considérée comme directe - atteignant d'emblée son objectif - que dans le premier cas. L'action des huchements qui freinent, stimulent ou réprimandent est indirecte (modifiant l'humeur de l'animal qui, par suite, modifie son allure), positive pour la plupart (ou négative, dans le cas du huchement de réprimande), toujours participative puisqu'elle n'a d'effet que si l'animal consent à y répondre. À la différence des cris d'appel des troupeaux, les aides vocales employées pour diriger les animaux de travail ne correspondent généralement pas à des leurres, car elles ne sont pas fondées sur une tromperie des 
sens de l'animal. La distinction est néanmoins ténue : certes, le trille est essentiellement un huchement impressif, par l'effet calmant du $r$ longuement roulé, mais elle n'est pas si éloignée du leurre, car elle ressemble aussi au bruit du cheval qui s'ébroue, attitude qui manifeste la détente chez l'équidé.

p.90 Si les huchements d'appel s'inspirent des cris de l'espèce concernée, les huchements qui visent à faire fuir les animaux ressemblent-ils aux cris de leurs prédateurs ? Ce n'est pas toujours le cas, tant s'en faut. N.Fijn signale cependant que les Mongols crient « $O-o-o-o b$ » pour attirer l'attention du bétail sur la possible présence de loups (2011). Par ailleurs, le $\hat{s}^{-} \hat{s}^{-} \hat{s}-\hat{s} i$ émis par les Touvas pour faire avancer les moutons rappelle le sifflement du serpent, mais aussi le bruit que font les moutons euxmêmes pour signaler un danger à leurs congénères, en même temps qu'ils tapent du sabot sur le sol (Darža, 2003 : 51). Dans ce cas, les hommes ne feraient, en fin de compte, que reprendre un leurre utilisé par les animaux eux-mêmes, qui combinent l'imitation d'un prédateur (le sifflement) et un bruit le faisant fuir (taper du pied sur le sol).

Par ailleurs, tous les leurres employés pour diriger les mouvements des animaux ne sont pas des huchements. Ainsi des gardiens de troupeaux peuvent appâter leurs bêtes en agitant ostensiblement un vieux sac de céréales vide, faisant espérer une distribution, espoir qui, s'il n'est pas trop souvent déçu, ne manquera pas de les faire venir.

Pour conclure temporairement sur cette question, nous remarquerons que les huchements de conduite du bétail ne répondent qu'imparfaitement à la logique du leurre. Si certains sont motivés dans leur forme par l'imitation du cri animal, il s'avère que, dans l'émission de ces huchements, les bergers apparaissent peu soucieux de parfaire cette imitation ${ }^{19}$. Aussi leur efficacité se fonde surtout sur la répétition, créant un conditionnement, ainsi que sur leur impressivité (brrr, ču) plus que sur leur expressivité. Les huchements des pâtres centrasiatiques sont plus proches d'onomatopées tendant vers la lexicalisation que des vocalises des chasseurs amazoniens, qui nécessitent, pour leur émission, une maitrise vocale et donc un apprentissage plus conséquents ${ }^{20}$.

\section{Quelques autres exemples de leurres}

L'animal lui-même peut être utilisé comme leurre dans la chasse, la monture servant de cachette au chasseur, voire d'appât pour faciliter l'approche du gibier (Ossendowski, 1996 [1925] : 243). En Iakoutie, le cheval sert non seulement à transporter le chasseur, mais aussi à le dissimuler, car les oies et les canards n'ont pas peur des chevaux ${ }^{21}$. Face au renne sauvage, c'est le renne domestique qui est le plus à même de jouer ce rôle (Hudâkov, 1969 : 67-69).

Enfin d'autres leurres sont adressés par les pasteurs centrasiatiques, non plus au bétail mais à ses prédateurs. C'est notamment le cas des épouvantails à loups mongols, kazakhs ou kirghizes. Leur effet est non tant visuel qu'odorant, car ils sont composés de vieux vêtements longtemps portés et de tissus enduits de poudre à fusil (Lescureux, 2007 : 194). Les éleveurs savent parfaitement prendre en

p.91 compte les capacités olfactives du canidé pour confectionner un leurre à sa mesure. La confiance en l'efficacité de ce stratagème est néanmoins limitée, puisqu'il ne dispense pas de la surveillance des troupeaux par des bergers et de l'emploi de chiens pour donner l'alarme. 


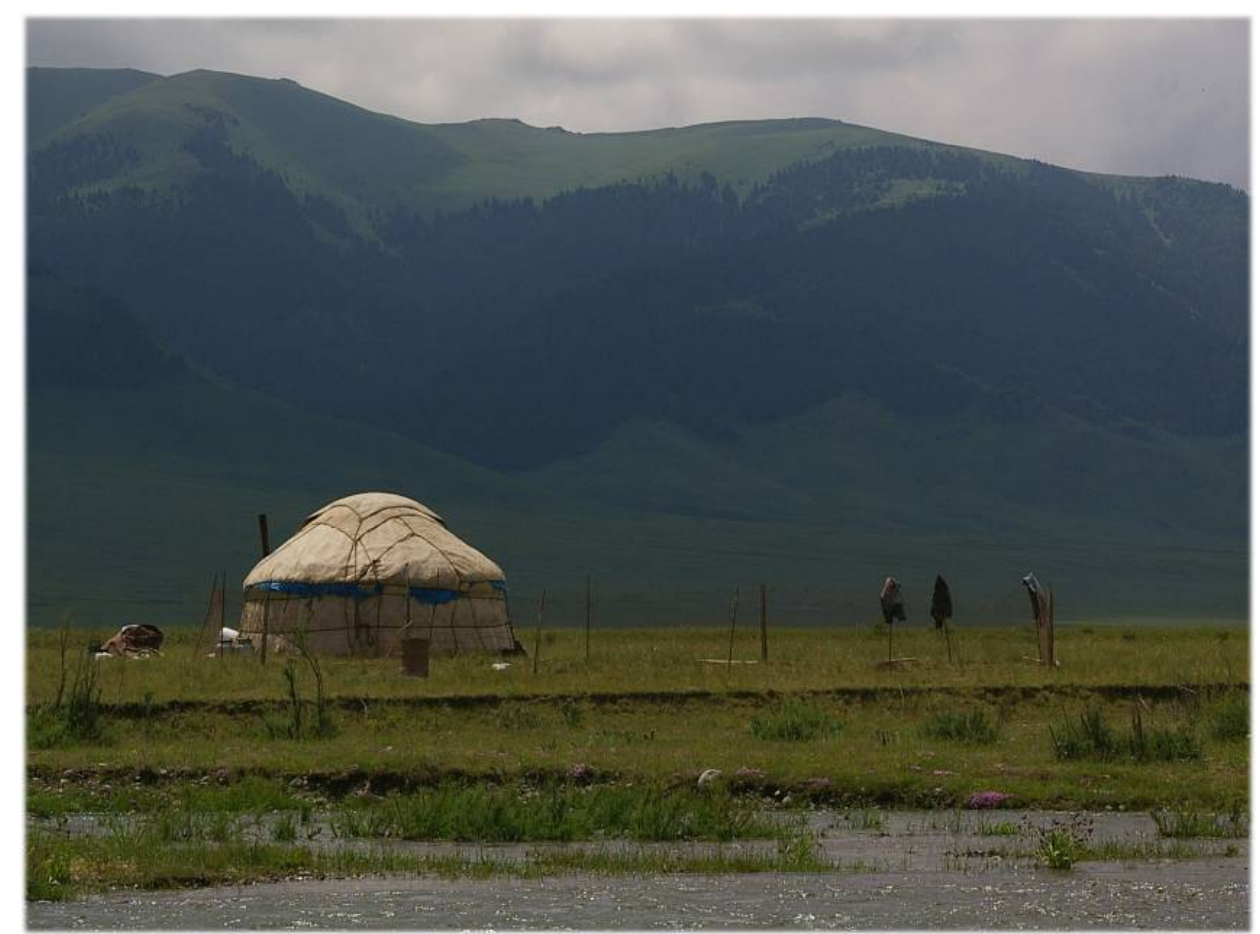

Vêtements posés sur les piquets de l'enclos à moutons faisant office d'épouvantails à loups (à droite de la iourte)

Photographie C.Ferret, Kazakhstan, région d'Almaty, district de Rajymbek, juin 2012

Les procédés décrits ici, pour inciter les femelles à donner leur lait ou pour diriger le bétail, ne correspondent donc que partiellement à la formule « faire croire pour faire agir » caractéristique du leurre. Leurrer la nature, oui, parfois. Mais la nature est-elle dupe ? Si ces techniques constituent indéniablement de beaux exemples de manipulation des bêtes, peut-on pour autant les considérer, sans craindre l'oxymore, comme de "véritables leurres » ? Jusqu'à quel point les juments croientelles que c'est leur poulain qui les tète quand il est à leurs côtés ? Dans quelle mesure le bétail croitil que c'est un congénère qui les appelle quand son maître émet une vocalisation inspirée par son cri ? Je me garderai bien de répondre à ces questions. En nous limitant strictement au point de vue humain - le seul qui puisse et doive être le nôtre, en tant qu'anthropologues ${ }^{22}-$, nous ne pouvons que constater que les acteurs, tout en sachant, grâce à leur longue expérience de familiarité avec les bêtes, adapter la forme du leurre aux capacités sensorielles des animaux, n'adhérent pas totalement à l'idée que ces derniers y croient.

\footnotetext{
${ }^{1}$ Le système de translittération du cyrillique utilisé dans cet article correspond à la norme ISO 9 de 1995 légèrement simplifiée pour les langues non slaves.

${ }^{2}$ Pour une présentation des formes d'actions, voir Ferret, 2012.

${ }^{3}$ Voir par exemple, pour les chamelles chez les Kazakhs, Dobrosmyslov (1895: 239-240); pour les vaches chez les Bouriates, Batueva (1992 : 19-20). Ici je décrirai principalement la traite des vaches et des juments, telle que je l'ai observée sur les terrains sibériens et centrasiatiques.

${ }^{4}$ Observation de terrain en Iakoutie, ulus d'Ust'-Aldan. En iakoute, la traite se divise en plusieurs moments appelés respectivement iak. usat "traite après la première sucée"; ètèt "traite après la deuxième sucée"; et même ùsùs (ynab ùsùsè) "traite après la troisième sucée", tandis que le verbe iak. ètèr désigne précisément le fait de laisser le veau téter un peu avant la traite (Pekarskij, 1907-1930 : 315, 3078, 3189).
} 
${ }^{5}$ Une technique alternative, consistant à pratiquer des injections d'ocytocine, a également été expérimentée (Langlois 1986 : 40).

${ }^{6}$ H.Serruys (1985 : 64) regrette que T.Chagdasüren, qui cite le même fait chez les Mongols (« Les chansons amadoyant la femelle qui refuse d'accepter son petit nouveau-né », Rocznik Orientalistyczny, XXXIII, 1, 1969, p. 97-102), ne fournisse aucune explication à ce sujet, mais il s'agit vraisemblablement de la même tactique.

${ }^{7}$ Pour plus de détails, voir l'article d'A.-M.Brisebarre dans le présent volume.

${ }^{8}$ F.Léotar (2006) a montré que les «mélodies huchées» - ou «chants de pâtres » (Pinon, 1967) - des éleveurs d'Asie intérieure, à savoir ces airs adressés au bétail comprenant des vocalisations spécifiques, partagent, de Touva à l'Ouzbékistan, un modèle musical similaire.

9 De même que d'autres pasteurs, cf., par exemple, Digard (1981:59) et plusieurs articles du numéro 84 de la revue Ethnozootechnie intitulé L'bomme et l'animal : voix, sons, musique... dirigé par M.Barboff et C.Griffin-Kremer (2009).

${ }^{10}$ D’après Middendorf (1869-1877, II : 545) ; Maak (1887 : 153) ; Seroševskij (1993 [1896] : 164) ; Maj (2007 : 166-167) ; mes observations de terrain dans les ulus d'Ust'-Aldan et de Verhoânsk entre 1994 et 2011 ; ainsi que, pour l'ensemble de ces tableaux : Dor $(1995$; 2003). Les huchements sont souvent répétés deux, trois fois, ou davantage, jusqu’à ce qu'ils soient suivis d'effet. Leur notation - parfois approximative et toujours imparfaite - a été adaptée au système de transcription utilisé dans l'article.

${ }^{11}$ D'après Dobrosmyslov $(1895: 43,47,91,95,183,185,233,271)$ pour la région de Turgaj ; mes observations de terrain dans le sud et l'est du Kazakhstan entre 1994 et 2012.

12 D’après Darža (2003: 50-51).

${ }^{13}$ D’après Bassanoff, 1975 : 129, citant Č. Sodnom, «Malyn tuxajt üg », Studia Mongolica, IV, 14, 1964, p.15-31; Levin \& Süzükei, 2011 [2006] : 137 et CD piste 33 pour les Mongols Dörvöd de la région d’Uvs, dans l'ouest de la Mongolie ; Fijn, 2011 : Vocalisation Dictionary pour la région d'Arkhangaï.

${ }^{14}$ Observation et entretien en mars 1994, ulus d'Ust'-Aldan, Iakoutie.

${ }^{15}$ R.Dor précise : «l'expressivité ne signalant pas pêle-mêle et confusément l'intensité et l'affectivité, mais dénotant une volonté d'instaurer la communication, témoignant de la volonté d'extériorisation du locuteur qui adopte le langage de son allocutaire » (2003: 392).

${ }^{16}$ Selon Dari Bandi, l'informateur dörvöd de T.Levin et V.Süzükei, le sifflement pour l'abreuvement, plus doux que les sifflements émis pour faire avancer un troupeau, serait une imitation, non de l'animal qui boit, mais du vent ; et les chevaux, qui prennent plaisir à entendre ce bruit, apprennent à l'associer à l'abreuvement (2011 [2006] : 137).

17 «Le linguiste danois K.Nyrop relevait en se gaussant que le canard français fait "coin-coin” alors que le canard danois fait "rap-rap" » (Dor, $2002:$ 133).

${ }^{18}$ Avant d'avoir vu le beau film d'A.Kurosawa, qui se douterait que Dode's Caden retranscrit le bruit du tramway ? Et pourtant, quand cette expression est répétée sur le bon rythme, la ressemblance phonétique est indéniable. Sur le caractère

p.93 à la fois motivé et conventionnel des onomatopées, $c f$. Saussure (1995 [1916] : 102).

${ }^{19}$ V.Süzükej souligne la remarquable capacité à imiter les cris des animaux domestiques et sauvages, acquise par les enfants des bergers touvas, lors des longues heures passées à garder le bétail, un talent particulièrement développé chez certains individus (Levin \& Süzükej, 2011 [2006] : 85]). Cependant, lorsque ces enfants apprennent à conduire les troupeaux, ne reproduisent-ils pas plutôt les huchements des adultes qu'ils ne cherchent à imiter les cris animaux ?

${ }^{20}$ Voir le texte d'Andréa-Luz Gutierrez Choquevilca dans le présent volume.

${ }^{21}$ Enquêtes personnelles de terrain en 1995 dans l'ulus de Srednekolymsk.

${ }^{22}$ Loin de manifester un pas en avant pour les sciences sociales, la tendance dominant actuellement dans de nombreuses études animalières, consistant à substituer l'anthropomorphisme à l'anthropocentrisme, risque de représenter une terrible régression.

\section{BIBLIOGRAPHIE}

Badamxatan, S.

1986 Les chamanistes du Bouddha vivant, monographie sur l'ethnie darxad de la province de Xövsgöl, Études mongoles et sibériennes 17.

Barmincev, Û.N., V.S.Kovešnikov, I.N.Nečaev \& al. 1980 Produktivnoe konevodstvo, Moskva, Kolos.

Baroin, C.

1975 «Techniques d'adoption en milieu animal (Daza du Niger)» in L'bomme et l'animal, premier colloque d'etbnozoologie, Paris, Institut international d'ethnosciences : 493-495. 
Bašarin, G.P.

1962 Istoriâ životnovodstva v Âkutii (vtoroj poloviny XIX-načala XX v.), Âkutsk, Âkutskoe knižnoe izdatel'stvo.

Baskin, L.M.

1982 «Behaviour Studies as a Basis for Horse Breeding Zootechnology », Production pastorale et société 11 : 29-44.

Bassanoff, N.

1975 «Adjurations, conjurations, un des aspects du pouvoir magique de la parole », Études mongoles 6 : 123-146.

Batueva, I.B.

1992 Burâty na rubeže XIX-XX vekov (Hozâjstvo burât. Skotovodstvo v dorevolûcionnoj period). Istorikoètnografičeskoj očerk, Ulan-Udè.

Bernus, E.

1980 "Vocabulaire relatif aux techniques d'adoption par les animaux en milieu touareg (Niger)», Journal des africanistes : 109-114.

2002 «Laits touaregs. Usages et symboles », in C.Raimond, E.Garine \& O.Langlois (ed.), Ressources vivrières et choix alimentaires dans le bassin du Tchad, Montpellier, IRD éditions : 399-412.

Burgat, F.

2004 "Non-Violence Towards Animals in the Thinking of Gandhi: The Problem of Animal Husbandry ", Journal of Agricultural and Environmental Ethics 14 : 223-248.

Čormanov, M.

1906 «Zametka o kirgizah Pavlodarskogo uezda », Zapiski Zapadno-Sibirskogo otdela RGO XXXII (6) : 1-30.

Darža, V.K. 2003 Lošad'v tradicionnoj praktike tuvincev-kočevnikov, Kyzyl, TuvIKOPR SO RAN.

Digard, J.-P. 1981 Techniques des nomades baxtyâri d'Iran, Cambridge - Paris, Cambridge University Press - ed. de la $\mathrm{MSH}$.

Dobrosmyslov, A.I.

1895 Skotovodstvo v Turgajskoj oblasti, Orenburg, Izdanie Turgajskogo oblastnogo statističeskogo komiteta.

Dor, R.

1982 «Une recherche en cours : les huchements du berger turc », Production pastorale et société 11 : 1321.

1993 «Les huchements du berger turc. II : Du huchement-aux-morts à l'appel des chevaux », Études turques et ottomanes $3: 27-41$.

1995 «Les huchements du berger turc. III : Interpellatifs adressés au gros bétail » Turcica XXVII : 199222.

2003 «À la recherche d'un proto-langage: Analyse de quelques huchements turcs relatifs au petit bétail », Bulletin de la société linguistique de Paris 98 : 385-408.

2002 «À l'aube du cri : De l'homme à l'animal avant le partage du monde », Diogène 200 : 129-139.

Evans-Pritchard, E.E.

1994 [1937] Les Nuer. Description des modes de vie et des institutions politiques d'un peuple nilote, Paris, Gallimard.

Ferret, C.

2006 Techniques iakoutes aux confins de la civilisation altaïque du cheval. Contribution à une antbropologie de l'action, Thèse de doctorat en anthropologie sociale et ethnologie, Paris, EHESS.

2007 «Les Iakoutes, des chercheurs de chevaux », in Ethnozootechnie 80 : 51-62.

2009 Une civilisation du cheval. Les usages de l'équidé de la steppe à la taïga, Paris, Belin.

2012 "Vers une anthropologie de l'action. André-Georges Haudricourt et l'efficacité technique ", L'Homme 202 : 113-140. 
Fijn, N.

2011 Living with Herds. Human-Animal Coexistence in Mongolia, Cambridge - New York, Cambridge University Press [annexe: Vocalisation Dictionary consulté en avril 2012 sur http://wimeo.com/1347497].

Gabyšev, M.F.

1972 Izbrannye trudy. Âkutskoe konevodstvo. Ėkonomičeskie i organizacionnye osnovy konevodstva, Âkutsk, Âkutskoe knižnoe izdatel'stvo.

Gmelin, J.G.

1767 [1751-1752] Voyage en Sibérie [...] fait aux frais du gouvernement russe, Paris, Desaint.

Hérodote

1990 [1964] L'enquête, Paris, Gallimard.

L'bomme

2009 L’homme et l'animal : voix, sons, musique..., Ethnozootechnie 84.

Hudâkov, I.A.

1969 Kratkoe opisanie Verboânskogo okruga, Leningrad, Nauka.

p.95 Huston Edgar, J.A

1924 «Strange Milking Custom », Man 24 : 39.

Jacquesson, S.

2000 Les oiseaux de chasse en Asie centrale : savoirs et pratiques, Thèse de doctorat, Paris, INALCO.

Langlois, B.

1986 L'élevage du cheval en Union Soviétique. Rapport technique faisant suite à une mission effectuée du 9 au 21 janvier 1984, Paris, INRA [Bulletin technique du département de génétique animale 40].

Léotar, F.

2006 "Les mélodies huchées des Touvas et des Ouzbeks. Contexte et système musical ", Études mongoles et sibériennes, centrasiatiques et tibétaines 36-37 : 351-372.

Le Quellec, J.-L.

2011 "Provoking lactation by the insufflation technique as documented by the rock images of the Sahara », Anthropozoologica 46(1) : 65-125.

Lescureux, N.

2007 Maintenir la réciprocité pour mieux coexister ? Ethnographie du récit kirghiz des relations dynamiques entre les bommes et les loups, Thèse de doctorat en ethno-écologie, Paris, Muséum national d'histoire naturelle.

Levin, T. \& V.Süzükei

2006 Where rivers and moutains sing: Sound, music and nomadism in Tuva and beyond, Bloomington, Indiana University Press.

Lienard, G. \& al.

2002 «Productivité de trois races bovines françaises, Limousine, Charolaise et Salers. Bilan de dix ans d'observations en exploitation », Productions animales 15 : 293-312.

Linhovojn, L.

s.d. [1972] "Le mode de vie des Bouriates de l'Aga avant la Révolution », in H.Larroche \& S.Popoff (ed.) Chamanes et lamas en Bouriatie, Paris, INALCO : 9-95.

Maak, R. K.

1887 Vilûjskij okrug Âkutskoj oblasti. III, Sankt-Peterburg, A.Tranŝel'.

Maj, E.

2007 Le cheval chez les Iakoutes chasseurs et éleveurs. De la monture à l'emblème culturel, Thèse de doctorat en anthropologie religieuse, Paris, EPHE.

Middendorf, A.F.

1869-1877 Putě̌estvie na sever i vostok Sibiri, II, Sankt-Peterburg Tipografiâ Imperatorskoj AN. 
Ossendowski, F.

1996 [1925] Asie fantôme. À travers la Sibérie sauvage. 1895-1905, Paris, Phébus.

Pekarskij, 亡̀.K.

1907-1930 Slovar' âkutskogo âzyka, Sankt-Peterburg - Petrograd - Leningrad, Tipografiâ imperatorskoj AN - Izdanie Rossijskoj AN - Izdanie AN SSSR.

Pinon, R.

1967 « Philologie et folklore musical. Les chants de pâtres avant leur émergence folklorique », Jabrbuch für Volksliedforschung 12 : 141-172.

Rubrouck, G. de

1985 Voyage dans l'empire mongol (1253-1255), Paris, Payot.

Saussure, F. de

1995 [1916] Cours de linguistique générale, Paris, Payot.

Seroševskij, V. L.

1993 [1896] Âkuty. Opyt ètnografičeskogo issledovaniâ, Moskva, Rossijskaâ političeskaâ ènciklopediâ.

Serruys, H.

1985 « Music and songs for animals », Études mongoles et sibériennes 16 : 61-67.

Šubskaâ, E.I. \& F.I.Saltykov

1931 Âkutskij krupnyj rogatyj skot, Leningrad, Izdatel'stvo AN SSSR.

Troŝanskij V.F.

1911 Nabroski o âkutab Âkutskogo okruga, Kazan', Tipo-litografiâ Imperatorskogo universiteta.

Tschinag G.

1996 [1994] Ciel bleu. Une enfance dans le baut Altä̈, Paris, Métailié.

\footnotetext{
Résumé

Leurrer la nature : quelques exemples de manipulation des bêtes en Asie intérieure

Leurrer est une manipulation de type "faire croire pour faire agir». Son efficacité se fonde sur une tromperie des sens. Elle implique que le sujet leurrant suppose une forme d'intentionnalité chez l'objet leurré. Outre la chasse au vol, domaine primordial du leurre, l'élevage pastoral en Sibérie et en Asie centrale nous fournit plusieurs exemples de telles manipulations. Ainsi, la méthode de la «sucée » employée pour la traite des femelles, avec participation du petit, montre que la mulsion n'est pas une simple opération de prélèvement, mais plutôt une manipulation de la femelle qui, abusée par la vue, l'odeur et le toucher de son petit, consent à donner son lait. De même, plusieurs procédés olfactifs, gustatifs, visuels, tactiles et auditifs (recouvrir le jeune adopté par la peau du petit mort, l'enduire de sel, des sécrétions vaginales de la mère, jouer de la musique, etc.) sont utilisés cas de refus d'allaitement de son petit ou d'adoption d'un autre. Pour le gardiennage, les huchements d'appel et de conduite des troupeaux ne répondent que partiellement à la logique du leurre. Si quelques-uns sont motivés dans leur forme par l'imitation du cri animal, il s'avère que, dans l'émission de ces huchements, les bergers apparaissent peu soucieux de parfaire cette imitation et leur efficacité se base surtout sur la répétition, créant un conditionnement. D’autres leurres, enfin, sont utilisés dans la chasse ou pour effaroucher les prédateurs du bétail, tels que les épouvantails à loups.
} 